\title{
Novel application of trimethyl chitosan as an adjuvant in vaccine delivery
}

This article was published in the following Dove Press journal: International Journal of Nanomedicine

\author{
Anshu Malik* \\ Manish Gupta* \\ Vatika Gupta \\ Himanshu Gogoi \\ Rakesh Bhatnagar \\ Molecular Biology and Genetic \\ Engineering Laboratory, School of \\ Biotechnology, Jawaharlal Nehru \\ University, New Delhi, India \\ *These authors contributed equally \\ to this work
}

\begin{abstract}
The application of natural carbohydrate polysaccharides for antigen delivery and its adjuvanation potential has garnered interest in the scientific community in the recent years. These biomaterials are considered favorable candidates for adjuvant development due to their desirable properties like enormous bioavailability, non-toxicity, biodegradability, stability, affordability, and immunostimulating ability. Chitosan is the one such extensively studied natural polymer which has been appreciated for its excellent applications in pharmaceuticals. Trimethyl chitosan (TMC), a derivative of chitosan, possesses these properties. In addition it has the properties of high aqueous solubility, high charge density, mucoadhesive, permeation enhancing (ability to cross tight junction), and stability over a range of ionic conditions which makes the spectrum of its applicability much broader. It has also been seen to perform analogously to alum, complete Freund's adjuvant, incomplete Freund's adjuvant, and cyclic guanosine monophosphate adjuvanation, which justifies its role as a potent adjuvant. Although many review articles detailing the applications of chitosan in vaccine delivery are available, a comprehensive review of the applications of TMC as an adjuvant is not available to date. This article provides a comprehensive overview of structural and chemical properties of TMC which affect its adjuvant characteristics; the efficacy of various delivery routes for TMC antigen combination; and the recent advances in the elucidation of its mechanism of action.
\end{abstract}

Keywords: trimethyl chitosan, chitosan, vaccine delivery, adjuvant, polymer

\section{Introduction}

The word adjuvant originates from the Latin dictionary "adjuvare," which means "to aid." The adjuvants are meant to enhance the immunogenicity of an antigen and thus reduce the number and amount of antigen doses to be administered. Some adjuvants help in the retention of the antigen in the host system and thereby help in improving the choice of routes for antigen delivery. Although there is no ideal definition for adjuvant, there are certain criteria that define good adjuvant properties. Bioavailability, no cytotoxicity, biodegradability, inexpensive production, no immune response against itself, appropriate response against the disease, for example, Th1 against tuberculosis and Th1/Th2 mixed against anthrax, suitability for the desired route of vaccination are the foremost to mention.

The live attenuated organisms and inactivated pathogens comprise several natural adjuvants, hence can elicit a robust immune response. The subunit and recombinant antigen-based vaccines often require adjuvants to augment the specific immune response against the corresponding antigen. The aluminum-based adjuvants are predominant to date and the only ones licensed for human use. Unfortunately, often with its potent adjuvant effect, accompanies several adverse side effects like local pain and inflammation, necrosis, granulomas, and ulcers and many systemic side effects
Correspondence: Rakesh Bhatnagar BSL-3, Molecular Biology and Genetic Engineering Laboratory, School of Biotechnology, Jawaharlal Nehru University, New Delhi I I0067, India Tel +9l II 26704079

Email rakeshbhatnagar@jnu.ac.in 
like nausea, fever, arthritis, uveitis, eosinophilia, allergy, anaphylaxis, organ-specific toxicity, and immunotoxicity. ${ }^{1-3}$ Aluminum is also concerned for its possible role in neurodegenerative diseases such Alzheimer's disease. ${ }^{4}$

The illustrious complete Freund's adjuvant (CFA) made by Freund in 1930, is an oil-in-water emulsion containing killed mycobacteria and is considered one of the most potent adjuvants. ${ }^{5}$ Its toxicity and local reactions make it unsuitable for human use. The incomplete Freund's adjuvant (IFA), however, is less toxic, being utilized in human vaccines. ${ }^{6}$ Similarly, several bacterial origin adjuvants were discovered later, such as lipopolysaccharide (LPS) and lipid A. ${ }^{7}$ Several bacterial DNA components have also been studied for their adjuvant potential, for instance, bacterial DNA containing CpG motifs, which are immunostimulatory and are regarded as a potent Th1 adjuvant. ${ }^{8}$ Over the time, a variety of adjuvants have been identified in the bacterial systems. Unfortunately, the toxicity of these adjuvants limits their use in humans. MF59 and adjuvant system 03 (AS03) are also oil-in-water emulsion-based adjuvants developed by Novartis and GlaxoSmithKline, respectively, and have been approved for pandemic influenza vaccines. ${ }^{9}$ However, a thorough characterization of both these formulations is still lacking. Virosomes containing a viral envelope, membrane lipids, glycolipids, but no genetic material have been proved to be excellent antigen-delivering agents and adjuvants. ${ }^{10,11}$ Similarly, immune stimulating complexes comprised saponin, cholesterol, phospholipid, and QS21 being the primary immunostimulant and are also currently in clinical trials for influenza, human papillomavirus and hepatitis $\mathrm{C}$ virus. ${ }^{12-14}$ Development of new adjuvants is necessary to help antigens become effective vaccines by inducing robust and enduring protective immune responses. New vaccine targets will require not only strong antibody responses but also robust cell-mediated immunity (CMI) including T helper (Th) cells and cytotoxic T lymphocytes. Alum alone will be inadequate for such cases because it poorly stimulates T-cell responses. Hence, there is a great need for the exploration of new adjuvants which could replace alum in future and have better properties. Since the initial trials for new adjuvants begin in small animal models, which cannot guarantee its direct translation in humans, it takes much time to test the potency of adjuvants and release them for human use. Moreover, since the vaccines are evolving and new route of immunization are being assessed, there is a demand for suitable and safer alternatives for adjuvants.

\section{Natural polymers as adjuvants}

Natural carbohydrate polysaccharides are new on the track of being investigated for their antigen delivery as well as adjuvant potential. The biomaterials due to their desirable properties like enormous bioavailability, non-toxicity, biodegradability, stability, inexpensive production, and immunostimulating ability are considered favorable candidates for adjuvant development. Table 1 represents the polysaccharides which have been investigated with various antigens and have been proven to possess the immune-modulating ability. For instance, inulin, hyaluronic acid, and alginate have also gained attention in the field of vaccine delivery. ${ }^{15-18}$ Glucans, dextrans, xylans, galactomannans, and glucomannans are also few polysaccharides that have been reported to possess immunomodulatory properties. ${ }^{19,20}$ Advax is the successful example of polysaccharide delta inulin-based adjuvant, which is currently undergoing a clinical trial. ${ }^{21}$ Chitosan is one such natural polymer, which has been appreciated for its antacid, antiulcer, and wound healing property, thus finds excellent applications in pharmaceuticals. ${ }^{22,23}$ Biocompatibility, biodegradability, no cytotoxicity, high charge density, low cost, mucoadhesive, permeation enhancing (ability to cross tight junction), and immunomodulating ability are the excellent properties of chitosan that makes the spectrum of its applicability much broader. ${ }^{24,25}$ Chitin (precursor of chitosan) and its derivatives have also shown promising results in the application of the development of cancer vaccine. ${ }^{26}$

\section{Chitosan: derivative of chitin, the second most abundant polysaccharide}

The polysaccharide chitosan [ $\alpha-(1-4)-2$-amino-2-deoxy- $\beta$-dglucan] is derived from chitin [(1-4)-2-acetamido-2-deoxy$\beta$-d-glucan] by its deacetylation. Chitin is the significant component of the body of all crustaceans and fungal cell wall. It has been extensively studied for its biomaterial property and use in various areas such as drug delivery, cosmetics, vaccine, antibacterial, and antifungal procedures. The insolubility of the chitosan in water makes it vulnerable to modifications that make it soluble and stable at a range of $\mathrm{pH}$. The chitosan dissolution in water is also possible by lowering the $\mathrm{pH}$ to slightly acidic $(\mathrm{pH}<5)$. The presence of several facile modifiable groups on the polymer (Figure 1) makes it suitable for chemical alteration, thereby changing its properties such as solubility and charge and making it readily employable for various applications. They have been particularly studied for their excellent mucoadhesive properties and permeation improvement applications in drug 
Table I A summary of the current status of research on the polysaccharide adjuvants

\begin{tabular}{|l|l|l|l|}
\hline Polysaccharide adjuvants & Source & Current status & Reference \\
\hline Inulin & Compositae & Clinical trial (Advax) & 21 \\
\hline Chitosan and its derivatives & Crustaceans and fungi & Preliminary research & 34 \\
\hline Galactomannan & $\begin{array}{l}\text { Caesalpinia spinosa, Aspergillus fumigatus, } \\
\text { Ramalina celastri, Morchella esculenta }\end{array}$ & Preliminary research & 113 \\
\hline Glucomannan & Candida utilis & Preliminary research & 114 \\
\hline Oligomannose & HIV, recombinant form obtained from bacteria & Preliminary research & 115 \\
\hline Dextran & Leuconostoc strains & Preliminary research & 116 \\
\hline Lentinan & Lentinus edodes & Preliminary research & 117 \\
\hline$\beta$-Glucans & Yeasts, seaweed, and algae & Preliminary research & 118 \\
\hline Zymosan & Saccharomyces cerevisiae & Preliminary research & 119 \\
\hline Lipomannan & Mycobacterium tuberculosis & Preliminary research & 120 \\
\hline Alginate & Brown algae & Preliminary research & 121 \\
\hline Levans & Aerobacter levanicum & Preliminary research & 122 \\
\hline
\end{tabular}

and protein delivery. Chitosan is evidently a non-toxic and biocompatible material. Consequently, the Food and Drug Administration (FDA) has approved the polymer for use in wound dressings and tissue engineering. ${ }^{27}$ The chitosanbased delivery studies are recent and could find potential applications in future. The various derivatives of chitosanlike glycated chitosan, thiolated chitosan, and trimethyl chitosan (TMC) are currently under investigation for drug delivery, vaccine delivery, and food-based applications.

Since chitosan is present naturally in the environment, there are several chitinases (hydrolyzing $N$-acetyl- $\beta-1,4-$ glucosaminide linkage) available in the environment, including plants and human, which can degrade the polymer, making its use environment-friendly. As the use of chitosan has increased in recent time, there is scope for its utilization in various applications such as a drug, DNA and antigen delivery, biosensor, imaging, and antifungal and antibacterial agent. Transport and delivery of vitamins (B6, B12, and C) have also been successfully tested by using TMC nanoparticles (NPs) as a carrier/vehicle. ${ }^{28}$

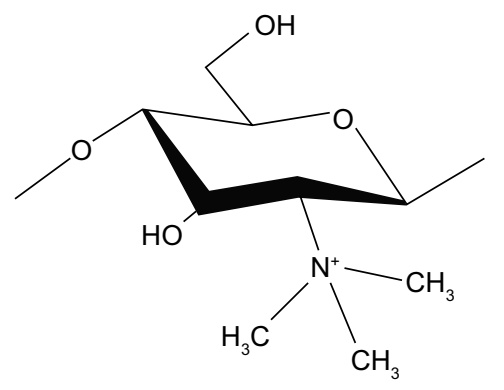

Figure I Chemical structure of trimethyl chitosan.

\section{Adjuvant properties of chitosan}

For a long time chitosan has been researched for its adjuvant properties, and here we have discussed some of its recent progress as an adjuvant. As chitosan bears mucoadhesive properties, it has frequently been explored via mucosal routes of administration. However, it seems suitable for parenteral delivery as well, as it performed comparably to the aluminum hydroxide in case of H5N1 influenza vaccination in a recent study. ${ }^{29}$ The chitosan NPs loaded with UV-inactivated dengue virus, and Bacillus of Calmette and Guerin cell wall components were able to stimulate the immature dendritic cells (DCs) and consequently caused maturation of these cells, proving its potential as an adjuvant candidate against dengue. ${ }^{30}$ Ovalbumin (OVA)/granulocyte-macrophage colony-stimulating factor-loaded chitosan NPs performed equivalently to IFA and CFA in the generation of OVA-specific antibody titer as well as $\mathrm{CD}^{+}$and $\mathrm{CD} 8^{+} \mathrm{INF}-\gamma^{+}$T cells. ${ }^{31}$

Chitosan has been tested with hepatitis B virus surface antigen $(\mathrm{HBsAg})^{32}$ diphtheria toxin, Mycobacterium tuberculosis CFP10-TB10.4 fusion protein, ${ }^{33}$ inactivated NIBRG-14 (H5N1) subunit antigen against influenza A, virus-like particles against norovirus, ${ }^{34}$ against poliovirus, ${ }^{35}$ against influenza A (H7N9, H9N2, H1N1, and H5N1) virus infection, ${ }^{36-38}$ and against Helicobacter pylori ${ }^{39}$ and has proved its potency as a successful adjuvant against these diseases. Gordon et al demonstrated that thermosensitive chitosan hydrogels possess higher ability to stimulate both humoral-mediated immunity and CMI, in addition to a slow and controlled in vitro release of antigen, compared to chitosan NPs, liposomes, and lipid-based cubosomes. 
Similarly, in an interesting study, chitosan-based hydrogel vaccines were illustrated to generate effective CD8 T-cell memory and performed equivalent to DC vaccination. ${ }^{40-43}$ Chitosan NPs are believed to augment the humoral and cellular immune response as well as able to elicit a balanced Th1/Th2 response. This makes them desirable for a broad range of therapeutic and prophylactic vaccines. ${ }^{44}$

\section{TMC: the highly desirable derivative of chitosan}

TMC is one of the highly desirable derivatives of chitosan and is preferred over chitosan due to its properties like high aqueous solubility and stability over a wide range of ionic conditions. Due to its mucoadhesive properties, ${ }^{45}$ it can open and cross the tight junctions of epithelial cells. ${ }^{46}$ Moreover, TMC forms polyplexes with DNA and has been utilized for gene delivery. ${ }^{47}$ An optimum degree of quaternization (DQ) is required to achieve transepithelial delivery of low molecular weight (MW) drugs and proteins, which has been found to be $40 \%-50 \%$. $^{48,49}$ These properties of TMC have been widely applied to its NP approach for delivering several antigens like OVA, tetanus toxoid, Omp19, Omp31, diphtheria toxoid, pDNA encoding antigen 85 of $M$. tuberculosis, Newcastle disease virus (NDV), whole inactivated virus (influenza), LTK63, and inactivated poliovirus. Table 1 summarizes the list of antigens that have been delivered with TMC as a mixture or encapsulated by TMC NPs.

\section{Synthesis strategies, properties, and characterization of TMC}

TMC is trimethylated chitosan and is largely derived from chitosan by reacting with iodomethane in stringent basic conditions upon solubilizing in $N$-methyl-2-pyrrolidone and using sodium iodide as a catalyst. ${ }^{50,51}$ It can also be derived by treating chitosan with suitable formaldehyde group compound to produce a Schiff- base, with subsequent reaction with a reducing agent and eventually with a methyl halide..$^{51,52}$ Reaction of chitosan with less poisonous and less expensive dimethylsulfate is a recent method being adopted. ${ }^{53}$ However, the resulting undesirable O-methylation occurs in almost all the reactions as they are carried out in strong basic conditions and high temperature which reduces the solubility of TMC in aqueous medium. To avoid this methyl iodide-based trimethylation could be carried out at lower temperature using dimethylformamide/ $\mathrm{H}_{2} \mathrm{O}$ mixture as solvent. ${ }^{54}$ Eschweiler-Clarke method followed by Verheul et al, is also another technique to obtain $O$-methyl free TMC. ${ }^{55}$ The final product TMC could be characterized for its trimethylation by performing H1-NMR after dissolving
TMC in $\mathrm{D}_{2} \mathrm{O}$. The DQ of TMC could then be measured from the NMR profile by using the following equation.

$$
\text { DQ }(\%)=\frac{\left[\left(\mathrm{CH}_{3}\right)_{3}\right]}{[\mathrm{H}]} \times 1 / 9 \times 100
$$

Here, $\left[\left(\mathrm{CH}_{3}\right)_{3}\right]$ is the integral of the hydrogens of the trimethylated amino groups at $3.3 \mathrm{ppm}$ and $[\mathrm{H}]$ is the integral of the H-1 peaks between 4.7 and 5.7 ppm in the NMR profile. Its MW could be determined using gel permeation chromatography. FTIR spectroscopy, CHN elemental analysis, 1 hour, and 13C NMR analysis can also be used for further characterization of its structure. The properties of TMC like aqueous solubility, mucoadhesion potential, and transepithelial movement is affected by its DQ. ${ }^{56}$

\section{Structural effect}

The adjuvanticity effect of TMC has been closely correlated with its DQ. According to Boonyo et al, a 40\% DQ of TMC is optimum for the immune response generation, as they tested with OVA intranasal administration. ${ }^{57}$ Similarly, with whole inactivated influenza virus (WIV) intranasal vaccination, a DQ of $37 \%$ was found to be better than $15 \%{ }^{58}$ The other factors that affect the adjuvant capability of TMC are its MW, the degree of acetylation (DAc) and the degree of O-methylation (DOM). Figure 1 clearly shows the modifiable functional groups of the polymer. It has been widely accepted that tailoring these three structural factors can lead to a differential immune response. ${ }^{57,58}$ The transport of WIV across Calu-3 cells was reduced by the reacetylated TMC, due to its rapid degradation relative to TMC. ${ }^{59}$ The DAc in case of chitosan has also been associated with its enzymatic degradation, ${ }^{60}$ permeation capacity, ${ }^{61}$ and antigen-presenting cell (APC) stimulation. ${ }^{62}$ Hence, DAc has a substantial effect on immune response elicitation. Regarding the MW of chitosan, Boonyo et al also reported that the immune response of the higher $\mathrm{MW}$ chitosan was better than the low MW variant. ${ }^{57}$ According to another study, the chitosan and TMC-based vaccine delivery via oral route with OVA were able to elicit an immune response against OVA but in a chitosan MW-dependent manner. ${ }^{63}$ Hence, an appropriate MW, as well as DQ and DAc, is required to achieve a suitable immune response. On the contrary, it was also demonstrated by a group that the DAc of $\leq 17 \%$ had a strong adjuvant effect which was not affected by the DOM or the DQ. ${ }^{64}$

Antigen conjugation with TMC has also been reported to boost the immune response in some instances. For example, 
the conjugation of the OVA with the TMC illustrated improved immunogenicity as well as uptake of the protein by DCs and consequently helped in the maturation of DCs. ${ }^{65}$ In a similar study, it was shown that the TMC-OVA conjugates were able to penetrate the skin more efficiently than the mixture of both and hence increased uptake by DCs of lymph nodes. ${ }^{66}$ Hence, the structural parameters affect the adjuvant capacity of TMC, and these could also be tailored to achieve a desired immune response.

\section{Route of administration}

The TMC adjuvanation effect also depends on the site of the administration. ${ }^{67} \mathrm{TMC}$ has an intrinsic mucoadhesive property, inherited from chitosan, as a result, nasal route has been explored the most. The TMC carries a positive charge at physiological $\mathrm{pH}$, and the nasal epithelium is negatively charged, this gives an extra edge for the electrostatic interaction of TMC with nasal epithelium. The nasal-associated lymphoid tissue allows the passage of TMC NPs conjugated proteins across it in addition to the increased immune response, upon nasal vaccination. ${ }^{68}$ The TMC NPs encapsulating OVA were able to increase the residence time of protein in the nasal epithelium and gave a high anti-OVA IgG as well as sIgA titer. These NPs were able to release the protein faster than the poly(lactic-co-glycolic acid) (PLGA) or PLGA/TMC NPs. ${ }^{69}$ In another attempt, it was seen that whole inactivated virus adjuvanted with TMC when immunized in the mice via nasal route did not produce any toxicity and was able to promote the uptake of the virus and amplify the immune response. $^{70}$ The tetanus toxoid-loaded TMC NPs intended for a nasal delivery were also prepared by few separate groups which showed quite good encapsulation efficiency as well positive surface charge suitable for nasal delivery as well as an enhanced immune response elicitation. ${ }^{71-74}$ According to another study, the intranasal administration of Escherichia coli enterotoxin LTK63 (mutant) with TMC in mice induced remarkable bactericidal titer in the mice model. ${ }^{75}$ Thus, the TMC NPs gave the researchers a direction to explore the nasal route of vaccination with the help of TMC-based vaccine formulations due to the virtue of its ability to cross nasal epithelium.

For oral delivery of an antigen, it has to be protected from the degradative conditions of the gastrointestinal tract. In an oral delivery study, the hepatitis surface antigen-loaded TMC NPs upon ionic gelation with hydroxypropyl methylcellulose phthalate, a $\mathrm{pH}$-sensitive polymer, protected the antigen from gastric destruction. ${ }^{76}$ According to another study, oral route delivery of the chitosan and TMC with
OVA were able to elicit an anti-OVA-specific immune response. ${ }^{63}$ Higher protection against Brucella melitensis and Brucella abortus infection was observed by oral route immunization in mice with TMC NPs encapsulating Omp19 as well as Omp31 than the intraperitoneal route. ${ }^{77,78}$ The intraduodenal route of administration was also verified by a group by administration of TMC NPs encapsulated OVA as antigen and led to significantly higher antibody rise than antigen alone. ${ }^{79}$

Several groups also explored the dermal route. TMC NPs encapsulating diphtheria toxin elicited quite high IgG titers, and the levels were similar to that of the alum upon intradermal injection. ${ }^{80}$ Utilizing the dermal or subcutaneous route, an innovative microneedle-based approach for vaccination was illustrated with the help of inactivated polio vaccine particles (negatively charged) and TMC (positively charged) by layering them onto $\mathrm{pH}$-sensitive microneedles and then immunizing rats. This approach led to the generation of antibodies against virus and emphasized the practical application of microneedles for dermal vaccination. ${ }^{81}$ In the same line, diptheria toxoid and TMC layering over the microneedles resulted in comparable immune response with the alum-adjuvanted diphtheria toxin upon dermal vaccination. ${ }^{82}$ Intramuscular immunization with pDNA encoding antigen $85 \mathrm{~A}$ of $M$. tuberculosis provoked a significant Th1 response in mice. ${ }^{83}$ Many routes have been explored for TMC vaccination. However, there is scope for further intervention into new routes.

\section{Particulate effect}

TMC NPs have gained recent interest as an adjuvant and a delivery vehicle for vaccination. The particles upon coencapsulation with an immunopotentiator further improves the strength and quality of immune response. In general, TMC NPs are prepared by the ionic gelation method, in which the positively charged TMC interacts with a negatively charged molecule and forms a complex. Even though researchers have tried many other alternatives to enhance the adjuvant effect of NPs, the highly used negatively charged molecule is tripolyphosphate (TPP). The TMC NPs prepared with LPS or MDP instead of TPP elicited higher levels of IgG and isotypes titer. ${ }^{84} \mathrm{CpG}$ as a negatively charged crosslinker has also been studied instead of TPP (conventionally used for TMC/TPP NP preparation), and it generated a strong Th1 as well as Th2 response upon nasal immunization. ${ }^{85}$ Chitosan NPs in combination with $\mathrm{C} 48 / 80$ mast cell activator also enhanced the immunogenicity of protective immunity of protective antigen. ${ }^{86}$ 
The MW of TMC polymer has not been reported to be a factor affecting the particle size or loading efficiency of NPs. However, the DQ has been found to be inversely associated with the size of the TMC NPs. ${ }^{87,88} \mathrm{TMC}$ particles within a size range of 200-300 $\mathrm{nm}$ were reported to be efficiently taken up by the DCs and also induced the activation of DCs. ${ }^{89}$ Similar sized vancomycin-loaded particles have been reported to demonstrate an efficient uptake by osteoblasts, resulting in their differentiation and elimination of the Staphylococcus aureus infection by controlled drug release. ${ }^{90}$ It has also been suggested that the smaller NPs are easily engulfed by the APCs in comparison with the large sized ones and could potentially be used as drug delivery agent against tumors. ${ }^{91}$ TMC NPs in the range 200-300 $\mathrm{nm}$ have also been reported to have more uptake as they are able to cross the nasal epithelial membrane owing to its small size. ${ }^{92}$ Recently, TMC NPs of $250 \mathrm{~nm}$ particle size encapsulating protective antigen were illustrated to possess adjuvant potential as well as provided protection against anthrax challenge in mouse model. ${ }^{93}$
Thus particle size also plays an important role in eliciting an immune response.

The TMC-based NPs are smaller in size in comparison with the chitosan NPs. Improved loading efficiency and release profile with a higher zeta potential makes TMC-based NPs much more desirable. ${ }^{94}$ Another study of TMC NPs encapsulating HBsAg reported that the protein was released for 43 days with no burst release with a remarkably higher loading efficiency of nearly $90 \% .{ }^{95}$

Several antigens have been tested with TMC NP-based vaccination and there has been a substantial evidence in support of its adjuvant potential. Table 2 lists the antigens, attenuated pathogens, and DNA that have been tested with the TMC NPs. For polio vaccination, virus-like particles coated with chitosan and TMC were tested for their stability and ability to interact with DCs. ${ }^{96}$ A robust humoral response was observed in a study where influenza matrix protein coding ectodomain fused with HSP70359-610 region of M. tuberculosis encapsulating TMC NPs was immunized intranasally

Table 2 A summary of antigens tested with TMC as an adjuvant

\begin{tabular}{|c|c|c|c|c|}
\hline TMC delivery form & Antigens & $\begin{array}{l}\text { Route of } \\
\text { immunization }\end{array}$ & $\begin{array}{l}\text { Animal } \\
\text { model }\end{array}$ & Reference \\
\hline Nanoparticles & Omp31 & Oral & Mice & 77 \\
\hline Nanoparticles & $\begin{array}{l}\text { Antigens of Newcastle disease } \\
\text { and infectious bronchitis }\end{array}$ & Intranasal & Chickens & 102 \\
\hline Microneedle layering & Diptheria toxoid & Dermal & Mice & 82 \\
\hline Nanoparticles & pDNA encoding antigen 85A & Intramuscular & Mice & 83 \\
\hline Nanoparticles & Newcastle disease viruses & Oral & Chickens & 101 \\
\hline Nanoparticles & $\begin{array}{l}\text { Unlipidated I } 9 \mathrm{kDa} \text { outer } \\
\text { membrane protein (U-Omp I9) }\end{array}$ & Oral, intraperitoneal & Mice & 78 \\
\hline Nanoparticles & OVA & Nasal & Mice & 106 \\
\hline TMC mixture & WIV & Intranasal & Mice & 59 \\
\hline $\begin{array}{l}\text { Microneedle layer (TMC mixture and } \\
\text { conjugated with antigen) }\end{array}$ & OVA & $\begin{array}{l}\text { Transcutaneous, } \\
\text { intradermal, intranodal }\end{array}$ & Mice & 17 \\
\hline Nanoparticles & OVA & Intradermal & Mice & 89 \\
\hline Nanoparticles & OVA & Intraduodenal & Mice & 79 \\
\hline Nanoparticles & OVA & $\begin{array}{l}\text { Intradermal, intramuscular, } \\
\text { intralymphatic }\end{array}$ & Mice & 67 \\
\hline Nanoparticles (CpG ionic crosslinker) & OVA & Nasal & Mice & 85 \\
\hline $\begin{array}{l}\text { Nanoparticles (mono-N-carboxymethyl } \\
\text { chitosan crosslinker) }\end{array}$ & Tetanus toxoid & Nasal & Mice & 74 \\
\hline TMC (varying DQ, DOM, molecular weight) & WIV & Nasal & Mice & 64 \\
\hline $\begin{array}{l}\text { Nanoparticles (mono-N-carboxymethyl } \\
\text { chitosan crosslinker) }\end{array}$ & Tetanus toxoid & Intranasal & Mice & 73 \\
\hline TMC mixture & LTK63 & Intranasal & Mice & 75 \\
\hline Microneedle layer & Inactivated polio virus & Dermal & Rats & 81 \\
\hline Nanoparticles (with alginate modification) & BSA & Subcutaneous & Mice & 123 \\
\hline
\end{tabular}

Abbreviations: BSA, bovine serum albumin; DOM, degree of O-methylation; DQ, degree of quaternization; OVA, ovalbumin; TMC, trimethyl chitosan; WIV, whole inactivated influenza virus. 
into the mice. This shows that the TMC NPs could also be an adjuvant and act as a vehicle for DNA vaccine delivery. ${ }^{97}$ Both chitosan and TMC NPs have been proved to be effective adjuvants in combination with Human T-lymphotropic virus-Type-I recombinant proteins env23 and env13 upon subcutaneous and intranasal immunization in mice. ${ }^{98}$ Against TB, pDNA encoding Ag85A and ESAT-6 protein encapsulated TMC NPs, generated a strong humoral response in mice and in case of ESAT-6, TMC NPs generated high levels of IgG2a and IFN-g, which are more critical for adequate protection against TB. ${ }^{99,100}$ Two recent studies reported on chickens with N-2-hydroxypropyl trimethyl ammonium chloride chitosan (N-2-HACC) NPs loaded with NDV and infectious bronchitis virus showed remarkable protection against the virus after oral immunization in chickens. ${ }^{101,102}$ In comparison with the alum-adsorbed $\mathrm{HB}$ and diphtheria toxin, TMC NPs loaded with HBsAg diphtheria toxin generated high IgG titers in both the cases and activated T-cell proliferation and produced a Th2-biased immune response in diphtheria vaccination. ${ }^{89,103}$ Similarly, the OVA-loaded TMC NPs generated a superior response to PLGA NPs when treated nasally into mice and also stimulated the maturation of DCs. $^{70}$ There are reports wherein the other nano-formulations coated with TMC also elicited a good immune response, and TMC aided in achieving that enhancement in the immune modulation. For instance, a long-term immune response was maintained upon the intranasal immunization with nanoliposomes coated with TMC, loaded with M-protein epitope of Group-A streptococcus. ${ }^{104}$ PLGA-based lipid polymeric particles as well as only PLGA NPs were coated with TMC and were observed to enhance the immunity. ${ }^{105,106} \mathrm{TMC}$ nanoformulations-based vaccine development is rising steadily, and it will soon become a preferred delivery vehicle and adjuvant among researchers.

\section{In vitro effect of TMC as an adjuvant}

According to previous studies, TMC stimulates the DCs and APCs and even caused the maturation and differentiation of these cells, simultaneously releasing several cytokines. For instance, Nantachit et $\mathrm{al}^{107}$ demonstrated that the in vitro stimulation of human nasal epithelial cells with TMC NPs encapsulating EDIII-D3 (domain III of dengue serotype-3 E protein) stimulated the secretion of several proinflammatory, Th1, and Th2 cytokines as well as chemokines. The particles uptake by DCs also upregulated the maturation markers (CD80, CD83, CD86, and HLA-DR) of DCs. ${ }^{107}$ Similar uptake and upregulation of maturation markers CD83, CD86, and $\mathrm{MHC}-\mathrm{II}$ was reported by a separate group. ${ }^{89}$ In another study, TMC-coated PLGA NPs and TMC-TPP NPs enhanced the antigen presentation by DCs, as shown by increased in vitro and in vivo $\mathrm{CD} 4{ }^{+} \mathrm{T}$-cell proliferation. ${ }^{106}$ Hence, the TMC NPs are efficiently captured by DCs which in turn stimulates the immune response.

Although the mechanism of action for the adjuvant effect of TMC is not entirely defined, it is hypothesized that it might possess a TRL recognition ability similar to chitin and thus could induce an innate immune response. ${ }^{108,109}$ A recent study by Carroll et al reveals some links of this mechanism of action. According to this study, the chitosan-induced maturation of DCs is mediated by the generation of type I interferons, eventually generating an antigen-specific Th1 response and $\mathrm{IgG} 2 \mathrm{c}$ rise. cGAS and STING (cytoplasmic DNA sensors) and mitochondrial ROS production are also reported to be involved in this Th1 response trigger. As chitosan has been linked to NLRP3 inflammasome, contributing to Th1 cell polarization, TMC is postulated to follow a similar mechanism of action. ${ }^{110,111}$ Figure 2 shows some of the already known pathways and mechanism of action of TMC or its NPs. Further investigation is required to identify the downstream signaling events responsible for the action of TMC or chitosan.

\section{Conclusion}

TMC, a natural polysaccharide-based polymer, is gaining recognition as a highly potential adjuvant and is being researched by many research groups around the globe. Lately, TMC has been promisingly used for mucosal immunization and other applications owing to its encouraging results. It has various advantageous properties such as being non-toxic, biodegradable, excellent biocompatibility, strong immune response enhancement and low reactogenicity. Although the current understanding of the mode of action of TMC is theorized by stimulation through the Toll-like receptor, still it needs further investigation to define its applicability in generating a desired immune response (Th1/Th2). Its mucoadhesive property could be further explored to generate a variety of vaccine formulations for parenteral administration. Furthermore, the gamut of routes for TMC vaccination need to be broadened to make it employable against more bacterial and viral pathogens. As the TMC NPs can cross the tight junctions of epithelial cell membranes, the nasal vaccination could potentially be evolved to a new level with the help of this adjuvant. The intrinsic factors of TMC, such as its structure, the DAc, the degree of methylation, and the MW, which significantly affect its adjuvant properties that need to be probed with more permutation and combinations 


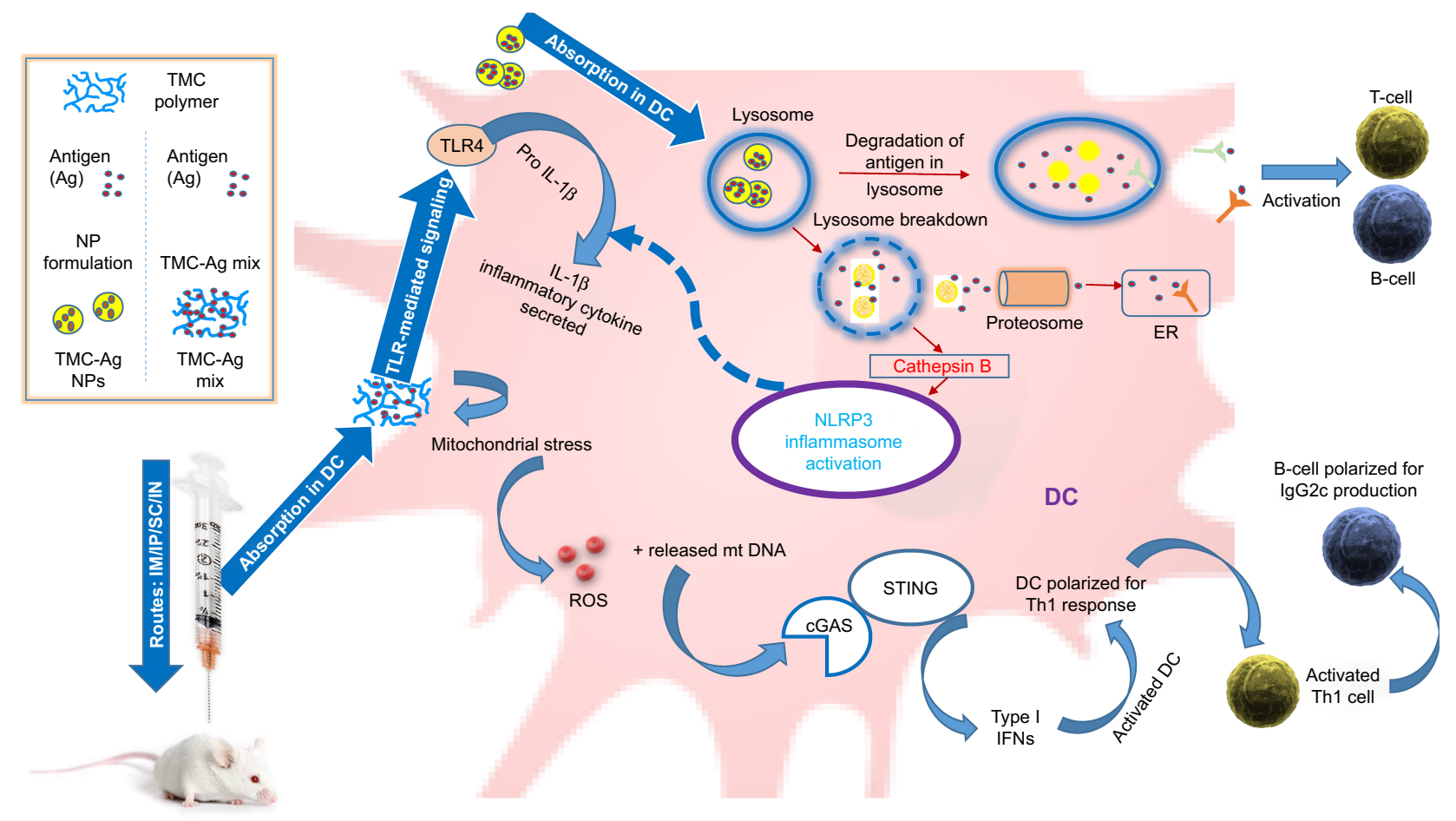

Figure 2 Mechanism of action of TMC as an adjuvant.

Abbreviations: DC, dendritic cell; ER, endoplasmic reticulum; IFN, interferon; IM, intramuscular; IN, intranasal; IP, intraperitoneal; NPs, nanoparticles; ROS, reactive oxygen species; SC, subcutaneous; TLR, Toll-like receptor; TMC, trimethyl chitosan.

to attain the best form of TMC as an adjuvant. The route of immunization and the form in which TMC (mixture, conjugated with antigen, NPs) is being used in combination with the antigen are also the decisive factors for an enhanced immune response generation. TMCs have been used for DNA delivery with some promising outcomes. Polyplexes formed by TMC and DNA interaction lead to condensation of the genetic material, which provides additional stability to the DNA. It also paves the way toward exploitation of TMC for DNA-based vaccines. Protection against various bacterial and viral pathogenic diseases also needs to be reconnoitered by employing TMC adjuvant-based vaccine strategies. In summary, TMC is a remarkably potential adjuvant for vaccine delivery and should be further investigated as an alternative to aluminum-based vaccines.

\section{Regulatory aspects and future outlook}

Chitosan and TMC possess many desirable properties like biocompatibility, less cytotoxicity, biodegradability, and mucoadhesiveness, and thus, they are one of the favorite candidates for vaccine delivery. Despite numerous vaccine studies in animal models have been reported using various antigens, chitosan and TMC have not yet been approved by the FDA as GRAS, hence could not be extended for human clinical studies for vaccination. ${ }^{112}$ Since chitosan has been approved by the FDA only for other topical applications like tissue engineering, wound healing, and cosmetic use, its approval for systemic applications would mark the next milestone in vaccine delivery. Therefore, further convincing studies are needed to emphasize the potential of TMC in numerous domains. The authors are hopeful that more vaccine studies demonstrating the already well-established functionality of TMC would aid in substantiating its use as an adjuvant in vaccine delivery.

\section{Acknowledgments}

We would like to thank all the members of the laboratory for thoughtful and lively discussions during this work. Author AM would like to thank the Council of Scientific and Industrial Research, India and Department of Biotechnology, India for providing financial support during this research term. We are thankful to the DST PURSE Program as well as the DBT Project "Upgradation of BSL-3, for studying dangerous pathogens with special references to Bacillus anthracis as well as to provide BSL-3 Facility to NER institutions and training of researchers of this region (sanction no: BT/55/ NE/2017)" for providing us financial aid.

\section{Disclosure}

The authors report no conflicts of interest in this work. 


\section{References}

1. Straw BE, MacLachlan NJ, Corbett WT, Carter PB, Schey HM. Comparison of tissue reactions produced by Haemophilus pleuropneumoniae vaccines made with six different adjuvants in swine. Can J Comp Med. 1985;49(2):149-151.

2. Bomford R. Aluminium salts: perspectives in their use as adjuvants. In: Gregoriadis G, Allison AC, Poste G, editors. Immunological Adjuvants and Vaccines. NATO ASI Series (Series A: Life Sciences), vol 179. Boston, MA: Springer; 1989:35-41.

3. Goto N, Kato H, Maeyama J, Eto K, Yoshihara S. Studies on the toxicities of aluminium hydroxide and calcium phosphate as immunological adjuvants for vaccines. Vaccine. 1993;11(9):914-918.

4. Mirza A, King A, Troakes C, Exley C. Aluminium in brain tissue in familial Alzheimer's disease. J Trace Elem Med Biol. 2017;40:30-36.

5. Freund J, Casals J, Hosmer EP. Sensitization and antibody formation after injection of tubercle bacilli and paraffin oil. Exp Biol Med. 1937;37(3):509-513.

6. Stuart-Harris CH. Adjuvant influenza vaccines. Bull World Health Organ. 1969;41(3):615-621.

7. Johnson AG. Enhancement of Antibody Response to Protein Antigens by A Lipopolysaccharide (Endotoxin) Derived from Salmonella Typhosa. College Park: University of Maryland; 1955.

8. Weiner GJ, Liu HM, Wooldridge JE, Dahle CE, Krieg AM. Immunostimulatory oligodeoxynucleotides containing the $\mathrm{CpG}$ motif are effective as immune adjuvants in tumor antigen immunization. Proc Natl Acad Sci U S A. 1997;94(20):10833-10837.

9. O'Hagan DT, Tsai TF, Brito LA. Emulsion based vaccine adjuvants. Hum Vaccin Immunother. 2013;9(8):1698-1700.

10. Moser C, Amacker M,Zurbriggen R. Influenza virosomes as a vaccine adjuvant and carrier system. Expert Rev Vaccines. 2011;10(4):437-446.

11. Moser C, Metcalfe IC, Viret JF. Virosomal adjuvanted antigen delivery systems. Expert Rev Vaccines. 2003;2(2):189-196.

12. Takahashi H, Takeshita T, Morein B, Putney S, Germain RN, Berzofsky JA. Induction of CD8+ cytotoxic $\mathrm{T}$ cells by immunization with purified HIV-1 envelope protein in ISCOMs. Nature. 1990;344(6269): 873-875.

13. He Z, Wlazlo AP, Kowalczyk DW, et al. Viral recombinant vaccines to the E6 and E7 antigens of HPV-16. Virology. 2000;270(1):146-161.

14. Polakos NK, Drane D, Cox J, et al. Characterization of hepatitis C virus core-specific immune responses primed in rhesus macaques by a nonclassical ISCOM vaccine. J Immunol. 2001;166(5):3589-3598.

15. Layton RC, Petrovsky N, Gigliotti AP, et al. Delta inulin polysaccharide adjuvant enhances the ability of split-virion $\mathrm{H} 5 \mathrm{~N} 1$ vaccine to protect against lethal challenge in ferrets. Vaccine. 2011;29(37): 6242-6251.

16. Mata E, Igartua M, Patarroyo ME, Pedraz JL, Hernández RM. Enhancing immunogenicity to PLGA microparticulate systems by incorporation of alginate and RGD-modified alginate. Eur J Pharm Sci. 2011;44(1-2):32-40.

17. Verheul RJ, Slütter B, Bal SM, Bouwstra JA, Jiskoot W, Hennink WE. Covalently stabilized trimethyl chitosan-hyaluronic acid nanoparticles for nasal and intradermal vaccination. J Control Release. 2011; 156(1):46-52.

18. Silva DG, Cooper PD, Petrovsky N. Inulin-derived adjuvants efficiently promote both Th1 and Th2 immune responses. Immunol Cell Biol. 2004;82(6):611-616.

19. Tizard IR, Carpenter RH, McAnalley BH, Kemp MC. The biological activities of mannans and related complex carbohydrates. Mol Biother. 1989;1(6):290-296.

20. Mata E, Salvador A, Igartua M, Hernández RM, Pedraz JL. Malaria vaccine adjuvants: latest update and challenges in preclinical and clinical research. Biomed Res Int. 2013;2013(24):1-19.

21. Gordon D, Kelley P, Heinzel S, Cooper P, Petrovsky N. Immunogenicity and safety of Advax $^{\mathrm{TM}}$, a novel polysaccharide adjuvant based on delta inulin, when formulated with hepatitis B surface antigen: a randomized controlled Phase 1 study. Vaccine. 2014;32(48): 6469-6477.
22. Ito M, Ban A, Ishihara M. Anti-ulcer effects of chitin and chitosan, healthy foods, in rats. Jpn J Pharmacol. 2000;82(3):218-225.

23. Dai T, Tanaka M, Huang YY, Hamblin MR. Chitosan preparations for wounds and burns: antimicrobial and wound-healing effects. Expert Rev Anti Infect Ther. 2011;9(7):857-879.

24. Xia Y, Fan Q, Hao D, Wu J, Ma G, Su Z. Chitosan-based mucosal adjuvants: Sunrise on the ocean. Vaccine. 2015;33(44):5997-6010.

25. Sinha VR, Singla AK, Wadhawan S, et al. Chitosan microspheres as a potential carrier for drugs. Int J Pharm. 2004;274(1-2):1-33.

26. Li X, Min M, Du N, et al. Chitin, chitosan, and glycated chitosan regulate immune responses: the novel adjuvants for cancer vaccine. Clin Dev Immunol. 2013;2013:387023.

27. Wedmore I, McManus JG, Pusateri AE, Holcomb JB. A special report on the chitosan-based hemostatic dressing: experience in current combat operations. J Trauma. 2006;60(3):655-658.

28. de Britto D, de Moura MR, Aouada FA, Mattoso LHC, Assis OBG. N,N,N-trimethyl chitosan nanoparticles as a vitamin carrier system. Food Hydrocoll. 2012;27(2):487-493.

29. Chang H, Li X, Teng Y, et al. Comparison of adjuvant efficacy of chitosan and aluminum hydroxide for intraperitoneally administered inactivated influenza H5N1 vaccine. DNA Cell Biol. 2010;29(9): 563-568.

30. Hunsawong T, Sunintaboon $P$, Warit S, et al. Immunogenic properties of a BCG adjuvanted chitosan nanoparticle-based dengue vaccine in human dendritic cells. PLoS Negl Trop Dis. 2015;9(9):e0003958.

31. Noh KH, Park YM, Kim HS, et al. GM-CSF-loaded chitosan hydrogel as an immunoadjuvant enhances antigen-specific immune responses with reduced toxicity. BMC Immunol. 2014;15:48.

32. AbdelAllah NH, Abdeltawab NF, Boseila AA, Amin MA. Chitosan and sodium alginate combinations are alternative, efficient, and safe natural adjuvant systems for hepatitis B vaccine in mouse model. Evid Based Complement Alternat Med. 2016;2016(1):7659684.

33. Yu W, Hu T. Conjugation with an Inulin-Chitosan Adjuvant Markedly Improves the Immunogenicity of Mycobacterium tuberculosis CFP10TB10.4 Fusion Protein. Mol Pharm. 2016;13(11):3626-3635.

34. Smith A, Perelman M, Hinchcliffe M. Chitosan: a promising safe and immune-enhancing adjuvant for intranasal vaccines. Hum Vaccin Immunother. 2014;10(3):797-807.

35. Ghendon Y, Markushin S, Akopova I, Koptiaeva I, Krivtsov G. Chitosan as an adjuvant for poliovaccine. J Med Virol. 2011;83(5):847-852.

36. Sui Z, Chen Q, Fang F, Zheng M, Chen Z. Cross-protection against influenza virus infection by intranasal administration of M1-based vaccine with chitosan as an adjuvant. Vaccine. 2010;28(48):7690-7698.

37. Sui Z, Chen Q, Wu R, et al. Cross-protection against influenza virus infection by intranasal administration of M2-based vaccine with chitosan as an adjuvant. Arch Virol. 2010;155(4):535-544.

38. Zheng M, Qu D, Wang H, et al. Intranasal administration of chitosan against Influenza A (H7N9) virus infection in a mouse model. Sci Rep. 2016;6:28729.

39. Gong Y, Tao L, Wang F, et al. Chitosan as an adjuvant for a Helicobacter pylori therapeutic vaccine. Mol Med Rep. 2015;12(3): 4123-4132.

40. Gordon S, Teichmann E, Young K, Finnie K, Rades T, Hook S. In vitro and in vivo investigation of thermosensitive chitosan hydrogels containing silica nanoparticles for vaccine delivery. Eur J Pharm Sci. 2010; 41(2):360-368.

41. Gordon S, Saupe A, McBurney W, Rades T, Hook S. Comparison of chitosan nanoparticles and chitosan hydrogels for vaccine delivery. J Pharm Pharmacol. 2008;60(12):1591-1600.

42. Gordon S, Young K, Wilson R, et al. Chitosan hydrogels containing liposomes and cubosomes as particulate sustained release vaccine delivery systems. J Liposome Res. 2012;22(3):193-204.

43. Highton AJ, Kojarunchitt T, Girardin A, Hook S, Kemp RA. Chitosan hydrogel vaccine generates protective CD8 T cell memory against mouse melanoma. Immunol Cell Biol. 2015;93(7):634-640.

44. Wen ZS, Xu YL, Zou XT, Xu ZR. Chitosan nanoparticles act as an adjuvant to promote both Th1 and Th2 immune responses induced by ovalbumin in mice. Mar Drugs. 2011;9(6):1038-1055. 
45. Snyman D, Hamman JH, Kotze AF. Evaluation of the mucoadhesive properties of $N$-trimethyl chitosan chloride. Drug Dev Ind Pharm. 2003; 29(1):61-69.

46. Kotzé AF, Luessen HL, de Leeuw BJ, de Boer BG, Verhoef JC, Junginger HE. N-trimethyl chitosan chloride as a potential absorption enhancer across mucosal surfaces: in vitro evaluation in intestinal epithelial cells (Caco-2). Pharm Res. 1997;14(9):1197-1202.

47. Mao Z, Ma L, Jiang Y, Yan M, Gao C, Shen J. N,N,N-Trimethylchitosan chloride as a gene vector: synthesis and application. Macromol Biosci. 2007;7(6):855-863.

48. Thanou MM, Kotzé AF, Scharringhausen T, et al. Effect of degree of quaternization of $\mathrm{N}$-trimethyl chitosan chloride for enhanced transport of hydrophilic compounds across intestinal caco- 2 cell monolayers. $J$ Control Release. 2000;64(1-3):15-25.

49. Hamman JH, Schultz CM, Kotzé AF. $N$-trimethyl chitosan chloride: optimum degree of quaternization for drug absorption enhancement across epithelial cells. Drug Dev Ind Pharm. 2003;29(2):161-172.

50. Sieval AB, Thanou M, Kotzé AF, Verhoef JC, Brussee J, Junginger HE. Preparation and NMR characterization of highly substitutedN-trimethyl chitosan chloride. Carbohydr Polym. 1998;36(2-3):157-165.

51. Domard A, Rinaudo M, Terrassin C. New method for the quaternization of chitosan. Int J Biol Macromol. 1986;8(2):105-107.

52. Kim CH, Choi JW, Chun HJ, Choi KS. Synthesis of chitosan derivatives with quaternary ammonium salt and their antibacterial activity. Polym Bull. 1997;38(4):387-393.

53. De Britto D, Assis OBG. A novel method for obtaining a quaternary salt of chitosan. Carbohydr Polym. 2007;69(2):305-310.

54. Öv R, Holappa J, Jónsdóttir S, Steinsson H, Másson M. N-selective 'one pot' synthesis of highly N-substituted trimethyl chitosan (TMC). Carbohydr Polym. 2008;74(3):740-744.

55. Verheul RJ, Amidi M, van der Wal S, van Riet E, Jiskoot W, Hennink WE. Synthesis, characterization and in vitro biological properties of O-methyl free N,N,N-trimethylated chitosan. Biomaterials. 2008;29(27):3642-3649.

56. Kulkarni AD, Patel HM, Surana SJ, Vanjari YH, Belgamwar VS, Pardeshi CV. N,N,N-Trimethyl chitosan: An advanced polymer with myriad of opportunities in nanomedicine. Carbohydr Polym. 2017;157:875-902.

57. Boonyo W, Junginger HE, Waranuch N, Polnok A, Pitaksuteepong T. Chitosan and trimethyl chitosan chloride (TMC) as adjuvants for inducing immune responses to ovalbumin in mice following nasal administration. J Control Release. 2007;121(3):168-175.

58. Hagenaars N, Mastrobattista E, Verheul RJ, et al. Physicochemical and immunological characterization of N,N,N-trimethyl chitosan-coated whole inactivated influenza virus vaccine for intranasal administration. Pharm Res. 2009;26(6):1353-1364.

59. Verheul RJ, Hagenaars N, van Es T, et al. A step-by-step approach to study the influence of $\mathrm{N}$-acetylation on the adjuvanticity of N,N,Ntrimethyl chitosan (TMC) in an intranasal nanoparticulate influenza virus vaccine. Eur J Pharm Sci. 2012;45(4):467-474.

60. Kean T, Thanou M. Biodegradation, biodistribution and toxicity of chitosan. Adv Drug Deliv Rev. 2010;62(1):3-11.

61. Schipper NG, Vårum KM, Artursson P. Chitosans as absorption enhancers for poorly absorbable drugs. 1: Influence of molecular weight and degree of acetylation on drug transport across human intestinal epithelial (Caco-2) cells. Pharm Res. 1996;13(11): 1686-1692.

62. Nishimura K, Nishimura S, Seo H, Nishi N, Tokura S, Azuma I. Macrophage activation with multi-porous beads prepared from partially deacetylated chitin. J Biomed Mater Res. 1986;20(9):1359-1372.

63. Boontha S, Junginger HE, Waranuch N, Polnok A, Pitaksuteepong T. Chitosan and trimethyl chitosan particles as oral vaccine delivery systems: comparison of the potential to initiate immune responses. JMMM. 2011;21(1):43-47.

64. Hagenaars N, Verheul RJ, Mooren I, et al. Relationship between structure and adjuvanticity of N,N,N-trimethyl chitosan (TMC) structural variants in a nasal influenza vaccine. $J$ Control Release. 2009;140(2):126-133.
65. Slütter B, Soema PC, Ding Z, Verheul R, Hennink W, Jiskoot W. Conjugation of ovalbumin to trimethyl chitosan improves immunogenicity of the antigen. J Control Release. 2010;143(2):207-214.

66. Bal SM, Slütter B, Jiskoot W, Bouwstra JA. Small is beautiful: $\mathrm{N}$-trimethyl chitosan-ovalbumin conjugates for microneedle-based transcutaneous immunisation. Vaccine. 2011;29(23):4025-4032.

67. Mohanan D, Slütter B, Henriksen-Lacey M, et al. Administration routes affect the quality of immune responses: a cross-sectional evaluation of particulate antigen-delivery systems. J Control Release. 2010; 147(3):342-349.

68. Liu Q, Zheng X, Zhang C, et al. Antigen-conjugated N-trimethylaminoethylmethacrylate chitosan nanoparticles induce strong immune responses after nasal administration. Pharm Res. 2015;32(1):22-36.

69. Slütter B, Bal S, Keijzer C, et al. Nasal vaccination with N-trimethyl chitosan and PLGA based nanoparticles: nanoparticle characteristics determine quality and strength of the antibody response in mice against the encapsulated antigen. Vaccine. 2010;28(38):6282-6291.

70. Hagenaars N, Mania M, de Jong P, et al. Role of trimethylated chitosan (TMC) in nasal residence time, local distribution and toxicity of an intranasal influenza vaccine. J Control Release. 2010;144(1):17-24.

71. Zarifpour M, Hadizadeh F, Iman M, Tafaghodi M. Preparation and characterization of trimethyl chitosan nanospheres encapsulated with tetanus toxoid for nasal immunization studies. Pharm Sci. 2013;18(4):193-198.

72. Chadhar V, Parashar AK, Patel M, Kumar R. Development and characterization of tetanus toxoid loaded trimethylchitosan chloride nanoparticles for nasal immunization. Current Research in Pharmaceutical Sciences. 2012;4:197-202.

73. Sayın B, Somavarapu S, Li XW, et al. Mono-N-carboxymethyl chitosan (MCC) and N-trimethyl chitosan (TMC) nanoparticles for non-invasive vaccine delivery. Int J Pharm. 2008;363(1-2):139-148.

74. Sayın B, Somavarapu S, Li XW, Sesardic D, Şenel S, Alpar OH. TMC-MCC (N-trimethyl chitosan-mono-N-carboxymethyl chitosan) nanocomplexes for mucosal delivery of vaccines. Eur J Pharm Sci. 2009;38(4):362-369.

75. Baudner BC, Morandi M, Giuliani MM, et al. Modulation of immune response to group $\mathrm{C}$ meningococcal conjugate vaccine given intranasally to mice together with the LTK63 mucosal adjuvant and the trimethyl chitosan delivery system. J Infect Dis. 2004;189(5):828-832.

76. Farhadian A, Dounighi NM, Avadi M. Enteric trimethyl chitosan nanoparticles containing hepatitis B surface antigen for oral delivery. Hum Vaccin Immunother. 2015;11(12):2811-2818.

77. Abkar M, Fasihi-Ramandi M, Kooshki H, Sahebghadam Lotfi A, Lotfi AS. Oral immunization of mice with Omp31-loaded $N$-trimethyl chitosan nanoparticles induces high protection against Brucella melitensis infection. Int J Nanomedicine. 2017;12:8769-8778.

78. Abkar M, Lotfi AS, Amani J, et al. Survey of Omp19 immunogenicity against Brucella abortus and Brucella melitensis: influence of nanoparticulation versus traditional immunization. Vet Res Commun. 2015;39(4):217-228.

79. Slütter B, Plapied L, Fievez V, et al. Mechanistic study of the adjuvant effect of biodegradable nanoparticles in mucosal vaccination. J Control Release. 2009;138(2):113-121.

80. Bal SM, Ding Z, Kersten GF, Jiskoot W, Bouwstra JA. Microneedlebased transcutaneous immunisation in mice with $\mathrm{N}$-trimethyl chitosan adjuvanted diphtheria toxoid formulations. Pharm Res. 2010;27(9):1837-1847.

81. van der Maaden K, Sekerdag E, Schipper P, Kersten G, Jiskoot W, Bouwstra J. Layer-by-layer assembly of inactivated poliovirus and $N$-trimethyl chitosan on $\mathrm{pH}$-sensitive microneedles for dermal vaccination. Langmuir. 2015;31(31):8654-8660.

82. Schipper P, van der Maaden K, Groeneveld V, et al. Diphtheria toxoid and $\mathrm{N}$-trimethyl chitosan layer-by-layer coated $\mathrm{pH}$-sensitive microneedles induce potent immune responses upon dermal vaccination in mice. J Control Release. 2017;262:28-36.

83. Poecheim J, Barnier-Quer C, Collin N, Borchard G. Ag85A DNA vaccine delivery by nanoparticles: influence of the formulation characteristics on immune responses. Vaccines (Basel). 2016;4(3):E32. 
84. Bal SM, Slütter B, Verheul R, Bouwstra JA, Jiskoot W. Adjuvanted, antigen loaded $\mathrm{N}$-trimethyl chitosan nanoparticles for nasal and intradermal vaccination: adjuvant- and site-dependent immunogenicity in mice. Eur J Pharm Sci. 2012;45(4):475-481.

85. Slütter B, Jiskoot W. Dual role of CpG as immune modulator and physical crosslinker in ovalbumin loaded N-trimethyl chitosan (TMC) nanoparticles for nasal vaccination. J Control Release. 2010;148(1):117-121.

86. Bento D, Staats HF, Gonçalves T, Borges O. Development of a novel adjuvanted nasal vaccine: $\mathrm{C} 48 / 80$ associated with chitosan nanoparticles as a path to enhance mucosal immunity. Eur J Pharm Biopharm. 2015;93:149-164.

87. Boontha S, Junginger HE, Waranuch N, Polnok A, Pitaksuteepong T. Formation of particles prepared using chitosan and their trimethyl chitosan derivatives for oral vaccine delivery: effect of molecular weight and degree of quaternization. Songklanakarin J Sci Technol. 2010;32(4):363-371.

88. Li XY, Li X, Kong XY, et al. Preparation of N-trimethyl chitosanprotein nanoparticles intended for vaccine delivery. $J$ Nanosci Nanotechnol. 2010;10(8):4850-4858.

89. Bal SM, Slütter B, van RietE, et al. Efficient induction of immune responses through intradermal vaccination with $\mathrm{N}$-trimethyl chitosan containing antigen formulations. J Control Release. 2010;142(3):374-383.

90. Xu J, Xu B, Shou D, Xia X, Hu Y. Preparation and evaluation of vancomycin-loaded N-trimethyl chitosan nanoparticles. Polymers 2015;7(9):1850-1870.

91. Song RF, Li XJ, Cheng XL, et al. Paclitaxel-loaded trimethyl chitosan-based polymeric nanoparticle for the effective treatment of gastroenteric tumors. Oncol Rep. 2014;32(4):1481-1488.

92. Amidi M, Romeijn SG, Borchard G, Junginger HE, Hennink WE, Jiskoot W. Preparation and characterization of protein-loaded $\mathrm{N}$-trimethyl chitosan nanoparticles as nasal delivery system. J Control Release. 2006;111(1-2):107-116.

93. Malik A, Gupta M, Mani R, Gogoi H, Bhatnagar R. Trimethyl chitosan nanoparticles encapsulated protective antigen protects the mice against anthrax. Front Immunol. 2018;9:562.

94. Boonyo W, Junginger HE, Waranuch N, Polnok A, Pitaksuteepong T. Preparation and characterization of particles from chitosan with different molecular weights and their trimethyl chitosan derivatives for nasal immunization. JMMM. 2017;18(2):59-65

95. Subbiah R, Ramalingam P, Ramasundaram S, et al. N,N,N-Trimethyl chitosan nanoparticles for controlled intranasal delivery of HBV surface antigen. Carbohydr Polym. 2012;89(4):1289-1297.

96. Tafaghodi M, Kersten G, Jiskoot W. Original Research Nano-adjuvanted polio vaccine: Preparation and characterization of chitosan and trimethylchitosan (TMC) nanoparticles loaded with inactivated polio virus and coated with sodium alginate. Nanomed J. 2014;1(4):220-228.

97. Dabaghian M, Ebrahimi SM, Nikbakhat Borojeni G. Use of N-trimethyl chitosan for intranasal delivery of DNA encoding M2e-HSP70c in mice. Iran J Vet Med. 2013;7(2):123-128.

98. Amirnasr M, Fallah Tafti T, Sankian M, Rezaei A, Tafaghodi M. Immunization against HTLV-I with chitosan and tri-methylchitosan nanoparticles loaded with recombinant env23 and env13 antigens of envelope protein gp46. Microb Pathog. 2016;97:38-44.

99. Karimi SM, Sankian M, Khademi F, Tafaghodi M. Chitosan (CHT) and trimethylchitosan (TMC) nanoparticles as adjuvant/delivery system for parenteral and nasal immunization against Mycobacterium tuberculosis (MTb) ESAT-6 antigen. Nanomed J. 2016;3(4):223-229.

100. Poecheim J. Adjuvanted Trimethyl Chitosan based Nanoparticle Formulations to Improve Immunogenicity of DNA Vaccines. Geneva: University of Geneva; 2015.

101. Zhao K, Sun Y, Chen G, et al. Biological evaluation of N-2-hydroxypropyl trimethyl ammonium chloride chitosan as a carrier for the delivery of live Newcastle disease vaccine. Carbohydr Polym. 2016;149:28-39.

102. Zhao K, Li S, Li W, et al. Quaternized chitosan nanoparticles loaded with the combined attenuated live vaccine against Newcastle disease and infectious bronchitis elicit immune response in chicken after intranasal administration. Drug Deliv. 2017;24(1):1574-1586.
103. Tafaghodi M, Saluja V, Kersten GF, et al. Hepatitis B surface antigen nanoparticles coated with chitosan and trimethyl chitosan: Impact of formulation on physicochemical and immunological characteristics. Vaccine. 2012;30(36):5341-5348.

104. Marasini N, Ghaffar KA, Giddam AK, et al. Highly immunogenic trimethyl chitosan-based delivery system for intranasal lipopeptide vaccines against group A streptococcus. Curr Drug Deliv. 2017;14(5):701-708.

105. Karavasili C, Bouropoulos N, Sygellou L, Amanatiadou EP, Vizirianakis IS, Fatouros DG. PLGA/DPPC/trimethylchitosan spraydried microparticles for the nasal delivery of ropinirole hydrochloride: in vitro, ex vivo and cytocompatibility assessment. Mater Sci Eng C Mater Biol Appl. 2016;59:1053-1062.

106. Keijzer C, Slütter B, van der Zee R, Jiskoot W, van Eden W, Broere F. PLGA, PLGA-TMC and TMC-TPP nanoparticles differentially modulate the outcome of nasal vaccination by inducing tolerance or enhancing humoral immunity. PLoS One. 2011;6(11):e26684.

107. Nantachit N, Sunintaboon P, Ubol S. Responses of primary human nasal epithelial cells to EDIII-DENV stimulation: the first step to intranasal dengue vaccination. Virol J. 2016;13(1):142.

108. Ishii KJ, Akira S. Toll or toll-free adjuvant path toward the optimal vaccine development. J Clin Immunol. 2007;27(4):363-371.

109. Schijns VE. Immunological concepts of vaccine adjuvant activity. Curr Opin Immunol. 2000;12(4):456-463.

110. Carroll EC, Jin L, Mori A, et al. The vaccine adjuvant chitosan promotes cellular immunity via DNA sensor cGAS-STING-dependent induction of type I interferons. Immunity. 2016;44(3):597-608.

111. Riteau N, Sher A. Chitosan: an adjuvant with an unanticipated STING. Immunity. 2016;44(3):522-524.

112. Bellich B, D’Agostino I, Semeraro S, Gamini A, Cesàro A. "The Good, the Bad and the Ugly" of Chitosans. Mar Drugs. 2016;14(5):E99.

113. Santander SP, Aoki M, Hernandez JF, et al. Galactomannan from Caesalpinia spinosa induces phenotypic and functional maturation of human dendritic cells. Int Immunopharmacol. 2011;11(6): 652-660.

114. Onishi N, Kawamoto S, Ueda K, et al. Dietary pulverized konjac glucomannan prevents the development of allergic rhinitis-like symptoms and IgE response in mice. Biosci Biotechnol Biochem. 2007;71(10):2551-2556

115. Ishii M, Kojima N. Mucosal adjuvant activity of oligomannosecoated liposomes for nasal immunization. Glycoconj J. 2010; 27(1):115-123.

116. McCarthy RE, Arnold LW, Babcock GF. Dextran sulphate: an adjuvant for cell-mediated immune responses. Immunology. 1977; 32(6):963-974.

117. Suzuki M, Takatsuki F, Maeda YY, Hamuro J, Chihara G. Lentinan: rationale for development and therapeutic potential. Clin Immunother. 1994;2(2):121-133.

118. Mirza Z, Soto ER, Dikengil F, Levitz SM, Ostroff GR. Beta-glucan particles as vaccine adjuvant carriers. Methods Mol Biol. 2017; 1625:143-157.

119. Ainai A, Ichinohe T, Tamura S, et al. Zymosan enhances the mucosal adjuvant activity of poly(I:C) in a nasal influenza vaccine. J Med Virol. 2010;82(3):476-484.

120. Leelayuwapan H, Kangwanrangsan N, Chawengkirttikul R, et al. Synthesis and immunological studies of the lipomannan backbone glycans found on the surface of Mycobacterium tuberculosis. J Org Chem. 2017;82(14):7190-7199.

121. Sarei F, Dounighi NM, Zolfagharian H, Khaki P, Bidhendi SM. Alginate nanoparticles as a promising adjuvant and vaccine delivery system. Indian J Pharm Sci. 2013;75(4):442-449.

122. Abraham E, Robinson A. Oral immunization with bacterial polysaccharide and adjuvant enhances antigen-specific pulmonary secretory antibody response and resistance to pneumonia. Vaccine. 1991;9(10):757-764.

123. Chen F, Zhang ZR, Yuan F, Qin X, Wang M, Huang Y. In vitro and in vivo study of N-trimethyl chitosan nanoparticles for oral protein delivery. Int J Pharm. 2008;349(1-2):226-233. 


\section{Publish your work in this journal}

The International Journal of Nanomedicine is an international, peerreviewed journal focusing on the application of nanotechnology in diagnostics, therapeutics, and drug delivery systems throughout the biomedical field. This journal is indexed on PubMed Central, MedLine, CAS, SciSearch $\AA$, Current Contents ${ }^{\circledR} /$ Clinical Medicine,
Journal Citation Reports/Science Edition, EMBase, Scopus and the Elsevier Bibliographic databases. The manuscript management system is completely online and includes a very quick and fair peer-review system, which is all easy to use. Visit http://www.dovepress.com/ testimonials.php to read real quotes from published authors.

Submit your manuscript here: http://www.dovepress.com/international-journal-of-nanomedicine-journal 